DEPARTAMENTO DE ZOOTECNIA GERAL, GENETICA ANIMAL E BROMATOLOGIA

Diretor: Prof. Dr. Milton de Souza Piza

DEPARTAMENTO DE QUIMLCA ORGANICA E BIOLOGICA

Diretor: Prof. Dr. Fonseca Ribeiro

\title{
SŌBRE OS MÉTODOS COLORIMÉTRICOS PARA A DETERMINAÇÃO DO FÓSFORO INORGÂNICO NO SANGUE DE EQUINOS. APLICABILIDADE E ESTUDO COMPARATIVO $\left({ }^{*}\right)$
}

\author{
(ABOUT TIIE COLORIMETRIC METHODS FOR THE DETERMINATION OF \\ INORGANIC PHOSPHORUS IN EQUINE BLOOD. APPLICABILITY AND \\ COMPARATIVE STUDY.
} Fernando Anireasi
Assistente
Virgílio BONOLDI

Livre Docente

Com o intúito de verificar quais os teôres normais de fósforo inorgânico no sangue de eqüinos, deparamos desde logo com uma dificuldade séria, qual seja, a da escolha de um método prático e acessível.

Dada a difícil aplicabilidade dos métodos gravimétricos, precisos, porém pou. co recomendáveis à prática diária, mercê da amostra exigida, propusemo-nos estudar os colorimétricos, largamente difundidos e preconizados para a determina. ção dêsse elemento no sôro de sangue humano.

Da leitura de alguns trabalhos orientados no sentido de estabelecer-se as taxas de fósforo e cálcio no sangue dos animais, deduz-se que os autores não se muniram da inicial precaução de verificar o método adotado. de cuja aplicabilidade não se pode confiar, senão depois de verificada experimentalmente.

Partindo desste ponto, dentre os diversos métodos colorimétricos que se têm à disposição para a dosagem dêsse elemento, no sangue humano, destacam-se os de Fiske-Subbarow $\left({ }^{1}\right)$, Benedict-Theis $\left({ }^{2}\right)$, Kuttner-Ljchtestein c outros, os quais encerram apreciável simplicidade, a par de grande accessibilidade.

Recaiu a escolha, dentro dessa ordem de idéias, nos métodos de Fiske-Subbarow, Benedict-Theis e R. S. Pereira $\left({ }^{3}\right)$.

\section{FUNDAMENTO QUAMICO DOS METODOS}

() fósforo sob forma de ion fosfato - em quantidades superiores à 1.2 mg por $100 \mathrm{~cm}^{3}$ — pode ser determinado de maneira prática c satisfatória por

(') Trabalho apresentado ao IV Congresso Brasileiro de Veterinária, realizado em janeiro de 1948, no Rio de Janeiro. 
Intermédio da formaçāo de anion fosfomolíbdico. A posterior redução e conse. qüente formação de um fosfoconjugado de molibdeno [4.(MoO3) MoO2] ${ }_{2} \mathrm{PO} 4 \mathrm{H} 3$ - fosfoconjugado cerúleo-molíbdico de Denigés - originará o aparecimento de côr azulada que se presta para fins colorimétricos ou fotométricos. Fntre os diversos redutores apropriados, citam-se o ácido 1-amino-2-naftol-4-sulfônico, a hidroquinona e a benzidina.

Baseiam-se na utilização dos dois primeiros redutores citados, os métodos do Fiske-Subbarow e de Benedict e Theis; o método de R. S. Pereira fornece ao meio, já préformado, o grupamento molibdoso, constituindo pois uma variante operatória.

\section{METODO DE FISKE-SUBBAROW}

$1.1-$ - Acido sulfírico $10 \mathrm{~N}$

Dissolver $\mathrm{cm}$ um béquer $278,5 \mathrm{~cm}^{3}$ de ácido sulfúrieo p.a., $\mathrm{D}=1,84$, $15 \circ \mathrm{C}$, en cêrca de $500 \mathrm{~cm}^{3}$ de água distilada; resfriar e transferir parra matraz aferich de um litro. Completar o volume.

1.2 - Molibdiato I (molibdato de amónio à $2,5 \%$ con ácido sulfúrico $5 \mathrm{~N}$ ).

Dissolver num béquer $12,5 \mathrm{~g}$ do sal, em circat de $100 \mathrm{~cm}$ a de ígua distiladia; transferir em seguicla para frasco volumitrico de $510 \mathrm{~cm}^{3}$; adlicionar 250 cnis de áciclo sulfúrico I0 N. Completar o volumc, após a lavargem do béquer.

1.3 - Molibdato II (molibriato de amonno à $2,5 \%$ en ácido sulfúrieno 3 N).

Proceder segundo acina referide para o molibelato $I$, rom a particularidade de juntar $150 \mathrm{~cm}^{3}$ de ácido sulfírico in $Y$

1.1 - Soluçño "Stock" de fosfato.

Dissolver ela cêrea de $100 \mathrm{mon}^{3}$ de água distiladil, proviamente sêco em

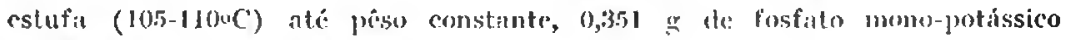
( I $\left.\left.^{2}\right)_{4} \mathrm{H}_{0} \mathrm{~K}\right)$, segundo surensen.

T'ransforir para um matra\% de um litro.

Juntar 10 cm: de ácido sulfurion 10 Ne completier a solume.

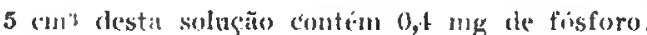

1.5 - Solıçัo picleño de fosfato.

Diluir à $104 \mathrm{~cm}^{\prime}$ num frasco volumetrico, $10 \mathrm{~cm}^{3}$ da soluçăo "stock" acima.

1.6 - Bissulfito de súdio a $15 \%$.

P'esar aproximalamente $30 \mathrm{~g}$ le bissulfito de sódio p.a., e dissolver ện cêréa de $200 \mathrm{~cm}^{3}$ de água distilada. Caso nõo se apresentar límpida, deixá-la em repouso pelo espaço de 2 à 3 dias antes de filtrar. 
1.7 - Sulfito de sódio a $20 \%$.

Pesar $200 \mathrm{~g}$ de sulfito de sódio $\left(\mathrm{SO}_{3} \mathrm{Na}_{2} \cdot 7 \mathrm{H}_{2} \mathrm{O}\right.$ p.a.) e dissolvê-las em cêrca de $380 \mathrm{~cm}^{5}$ de água distilada, filtrando se necessário. Guardar a solução em vidro de rôlha esmerilhada.

1.8 - Acido 1-amino--2-naftol-4-sulfônico, a $0,25 \%$.

Dissolver $0,25 \mathrm{~g}$ do ácido em $97,5 \mathrm{~cm}^{3}$ da solução de bissulfito de sódio a $15 \%$, adicionando $2,5 \mathrm{~cm}^{3}$ de sulfito de sódio a $20 \%$. Agitar até completa solubilização. Protegida do contacto do ar, a solução conserva-se cêrca de duas semanas.

1.9 - Kcirlo tricloroacético a $10 \%$.

Dissolver $50 \mathrm{~g}$ do ícido purissimo a $500 \mathrm{~cm}$, em ńgua distilada.

Auálise

a) Transferir para pequeno Lirlenuneyer $\left(50 \mathrm{~cm}^{3}\right) 8 \mathrm{~cm}^{3}$ de ácido tricloroacético a $10 \%$ e sob agitação constante, adicionar gotejando, $2 \mathrm{~cm}^{3}$ de sôro.

b) Filtrar, vertendo de uma só vez, em papel de filtro sêco e pràticamente isento de cinzas.

c) Pipetar $5 \mathrm{~cm}^{3}$ do filtrado e transferí-los para frasco volumétrico de $10 \mathrm{~cm}^{3}$ ou tubo graduado a $10 \mathrm{~cm}^{3}$.

d) Colocar en outro frasco ou tubo, $5 \mathrm{~cm}^{3}$ da solução padrão contendo 0,04 mg de fúsforo (1.5)

e) Acrescentar ao filtrado (c) I $\mathrm{cm}^{3}$ de toolibdato II (J.3) e ao padrão (d), $1 \mathrm{~cm}^{3}$ de molibdato I (1.2). Agitar ben.

f) Adicionar a ambos os tubos $0,4 \mathrm{~cm}^{3}$ do ácido amino-naftol-sulfónico (1.8) e completar o volume com água distilada. Homogenizar perfeitamente e proceder à leitura no colorimetro, após 5 minutos.

Cálculo

Para ser calculado o teor de fósforo, de posse dos dados fornecidos pelo colorimetro e das quantidacles utilizadas de sôro e filtrado, aplica-se a seguinte formula:

na qual

$$
\text { ang de fósforo em } 100 \mathrm{~cm}^{s} \text { de sôro }=\frac{100 \mathrm{Cc} . \text { Ac. } \mathrm{V}}{\text { Ad.F.S }}
$$

$\mathrm{Cc}=$ quantidade fósforo, em mg, existente no padrão;

$A c=$ altura em que ficou o prisma na solução padrão;

$\mathbf{V}=$ volume correspondente $\dot{a}$ sôma do ácido tricloroacético e sôro, pipetados na operaçũo de desalbuminizaçăo;

$\Lambda d=$ médias das leituras obtidas por comparação con o padrão;

$\mathbf{F}=$ volume do filtrado utilizado para o desenvolvimento da còr;

$S=$ sôro, $\mathrm{cm}^{\mathrm{cm}}$, tomado para desalbuminar. 
Notas:

O áciclo tricloroacélico utilizado na desalbuminização do sion deve ser de alta purezal, a fím de näo perturbar o desenvolvimento da côr. Além dissos, rerounchda-se fazer a pesquisa dos fosfatos. Esta procede-se dia seguinte mantira:

bispôr trís béqueres de forma natta, de $150 \mathrm{~cm}^{3}$ de capacidade, sôbre lundo branco.

Fin um dêles $(A)$, colocann-se $100 \mathrm{~cm}^{3}$ cie nigua. Noutro (B), juntant-sc 4hs

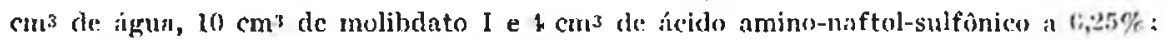
deverí resultar uma solução prätionente incolor; caso contrário, uma ou outrat das soluçōes adicionadas contém fosfato.

No teresiro béquer (C), adicionan-se. $10 \mathrm{~cm}^{3}$ de solução de ácido tricloroncillico, $4.5 \mathrm{~cm}^{3}$ de água, $10 \mathrm{~cm}^{3}$ de rnolibdato 11 e $\mathrm{cm}^{3}$ dat solução de icido amino-naftolsulfônico, agitanclo " conteńdo com anxilio de um bastĩo de vidlo. Caso năo se

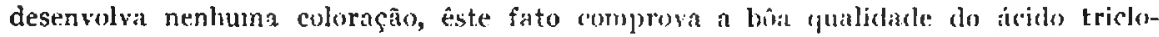
roacético utilizado na preparação da soluẹios: caso contrário, toma-se o béquer (Ił), e junta-se $1 \mathrm{~cm}^{3}$ da soluçaio de fosfato contendo $0,005 \mathrm{mg}$ por $\mathrm{cm}^{3}$, algitanclo cuidadosamente. Noras adições serĩo feitas, até que os conteúdos de ambos os béqueres apresentem a mesma coloração e isto a intervalos não menores a 2 minutos. $O$ volume da soluçăo de fosfato necessário parra conseguir-se a finalidade citadia, multiplicato por 0,05 darí a correşa $\mathrm{cm} \operatorname{lng}$ por $100 \mathrm{~cm}$, isto é, it quantidads it scr subtráda do resultado da análise do sangue.

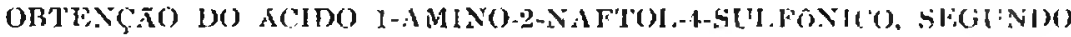 FUIIN (s)}

\section{Reagentes:}

Acito sulfúricu $₫ 10 \% \ldots \ldots \ldots \ldots \ldots \ldots \ldots \ldots, 1000 \mathrm{~cm}^{*}$

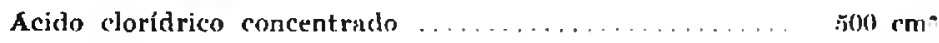

Solução a $10 \%$ de hidróxido de sódio .......... ; 390 (m)

Nitrito de sódio $\ldots \ldots \ldots \ldots \ldots \ldots \ldots \ldots \ldots \ldots \ldots . \quad 50 . \ldots \ldots$

Sulfito de sédio ........................ $50 \mathrm{~g}$

Bissulfito de sórdio ......................... $100 \mathrm{~g}$

Naftul-bet. ressublimads $\ldots \ldots \ldots \ldots \ldots \ldots \ldots \ldots, 100 \mathrm{~g}$

Proparaçâ:

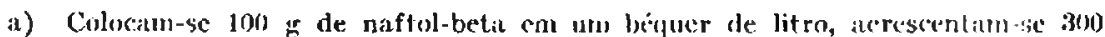

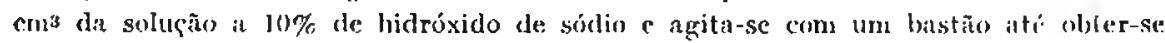
completa dissoluğ̃o (le 10 al 15 minutes).

b) Colocam-se de 50 in $55 \mathrm{~g}$ de nitrito de sódio con um béfuer de quattro litros; acrescentinu-se $600 \mathrm{~cm}^{3}$ de água e agita-se até il dissolução (de is at 5 minutos).

c) Verte-se a solução alcalina de naftol-beta em um brupuer de qualtu litros, com a soluçăo de nitrito e lava-se corn cêrca de $100 \mathrm{~cm}^{3}$ de ánua " primetiro béquer.

d) Acrescenta-se ì mistura unterior 800 gg de gêlo. 
e) Enche-se uma provetsa de $200 \mathrm{~cm}^{3}$ com soluçầ fria de ícido sulfirico a $10 \%$, vertendo-se pouco a pouco pelas paredes do béquer, enquanto sc agita com um bastão de vidro. Esta agitaç̃o devc prosscguir mais alguns minutos, apus se hatver adicfornado todo " ácido sulfúrico.

Re'pete-se a adiçä́n do ácido sulfúrico nas condiçöes acimat refericias, usando cada $10 \% 200 \mathrm{~cm}^{3}$ até atingir o volume de $800 \mathrm{~cm}^{3}$.

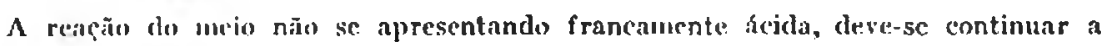

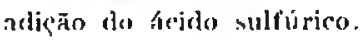

Lo juntar-se: o acido, comera a format-se um precjpitido amarclo, que aunenIa fradualmente, até converter tôda a mistura em una pasta semi-solida.

O procipitudo deve apresentar uma ligeira coloraçio esverdeada.

deixar an repouse, duraute uma hora, a fim de dissolver completamente o beta-naflat.

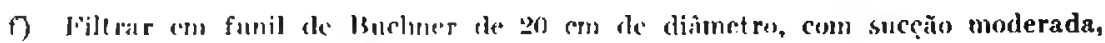

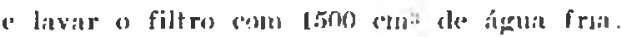

F) (olocar o precipitado beta-nitroso-naftol em una cipsula grande, pulveri\%ar sôbre cla $100 \mathrm{~g}$ de bissulfito de sódio e $50 \mathrm{~g}$ de sulfilo de sćclio e ageitar. Formn-se um composto de aliçäo com o bissulfito de śdio e a mistura lorna-se líquida. Filtrar imediatamente, em Buchner (de 12 a $15 \mathrm{~cm}$ de diametro), provido de papel de filtro duplo c re cinzas conhecidas: lavir em seguidit, com um pouco de igua distiladia.

h) Verte-se " filtrado imediatamente (para evitar um rxcessivo escurecimen(1) em um biefuer de ciuce litros ou en frisce, escuro de bôca larga, com $2000 \mathrm{~cm}^{3}$ de água e $500 \mathrm{~cm}^{3}$ ale ácido clorfalrico comentrado. Colocar um funil munido de

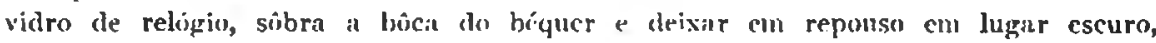
cerca de trinta e scis horas.

Decorrialo este tempo, notau-se no frasco furmaucoes gue tên o aspecto de agulhas brancas.

0 aparecimento de cristais escuros on rosialos dienota produtos de decomposição, devido à exposiçũo prolongada ì lu\%.

Filtrar em utu IBuchner nas conclip̧ies acima e lisvar conu dois litros de água distilada .

\section{PURIFICACAO DO ACIDO I AMINO-2-NAFTOL-4-SULFUNICO (6)}

1) - Purificagro pelo ilcoul: o produto final obtido, apos lavagens sucessivas com ugun, é tratado com álconl em quantidactes suficientes para descorar completamente os crislais.

2) - Purificagion por cristalizaçius: dissolver $150 \mathrm{~g}$ de bissulfito alc sódio e 10 Lf de sulfito de súdio rristaljado em un litro de iugua distilada. Adicionar $15 \mathrm{~g}$ de ícido-amino-niltol,sulfönico bruto, recim-preparado $c$ agilar. Is impurezas que o acompanlaun nho se solubilizan. A solução, depois de aquecida, filtrada atra-

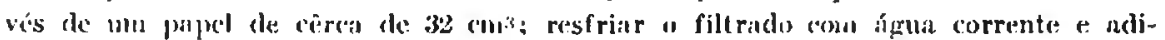
clemar 10 cma de ácido cloridrion concentrado. Ifiltrar com " aluxilio du trompa; lavir com cêrea de $300 \mathrm{~cm}^{3}$ de água $s$, finalmente, coun álcool, até o filtriklo apresentar-se incolor. O produto obtido deve ser seculo ato ar, sem exposigño a lez; a seguir, degwis de pulveri\%alo, guarda-se em vidro escuro. 


\section{METODO DE, BENEDICT-THEIS (2)}

Soluções reagentes:

2.1 - Acido sulfírico $(\mathrm{D}=1,84$ a $15 \circ \mathrm{C})$.

2.2 -- Solug̣ão de mulibrato de sódio, isento de amónio, a 8\%. Dissolver 20 de ácido molíbdico, isento de amônio, $\mathrm{em} 25 \mathrm{~cm}^{3}$ de uma soluçiio de hidróxidu de sódio a 20\%. Aquecer suavenente até dissoluçăo completa. Resfriar e transferir para um frasco volumétrico de 2 б̆o $\mathrm{cm}^{3}$, completanclo o volume com água distilada. Filtrar se necessário.

2.8 - Solução de hidroquinona a $0,5 \%$ em bissulfito de sódio a $15 \%$. Dissolver $15 \mathrm{~g}$ de bissulfito de sódio p.a., en cûrca de $50 \mathrm{~cm}^{3}$ de água distilada e acrescentar $0,5 \mathrm{~g}$ de hidroquinona $\mathrm{P}$. i..; transferir pari um matray aferido de $100 \mathrm{~cm}^{3}$, completando-se o volune com água distilada.

2.4 - Soluç̄̄o "stock" de fosfato monopotássico. Pesar, depois de séco em estufa $(110-115 \circ \mathrm{C}$ ), a pèso constante $0,351 \mathrm{~g}$ de fosfato mono-potíssico (POHH'LK), segundo Sörensen. Dissolver em um pouco de água distilada; transferir pura um frasco volumétrico de um litro, adicionando entåo $10 \mathrm{~cm}^{3}$ de ácicio sulfúrico $10 \mathrm{~N}$. Diluir, finalmente, à marca.

2.5 - Solução padrão de fosfato mono-potássico. Pipetur $10 \mathrm{~cm}$ " da soluçàu "stock" e diluir a $100 \mathrm{~cm}^{3}$ com agua distilada. $5 \mathrm{~cm}^{3}$ desta soluçãu encerram 0.04 mg de fósforo.

2.6 - Reagente molibdato-ácido sulfúrico. E' constituido de um volume da solução de molibdato (2.2) para um volume igual de ácido sulfúrico, $\mathrm{D}=1,8.1$ (2.1). Deve ser preparado no momento de usar.

2.7 - Acido tricloroacético a 10\%. Dissolver en água distiladit, usando frasco volumétrieo de $500 \mathrm{~cm}^{3}, 60 \mathrm{~g}$ de ácido puríssimo.

Andilise :

a) A precipitaça dos prótidos é obtida segundo técnica já descrita no inétodo anterior, guardada a relação de um volume de soiro para quatro de acido tricloroacético a $10 \%$.

b) Procedida a filtraçăo, segundo ji preconizadia, th em³ do filtrado sũo transportados para tubo innrcado a $10 \mathrm{~cm}^{3}$, ou frasco volumetrico dessa capacidude.

c) Em outro tubo on frasco, colocar $5 \mathrm{~cm}^{3}$ da soluçito padräo de fosfato (2.5).

d) Em seguida, adicionar a ambos, $3 \mathrm{~cm}^{3}$ de água distilada e $1 \mathrm{~cm}^{3}$ do reagente molibdato-ácido sulfúrico (2.6). Agitar cuidadosamente.

e) Acrescentar cntảo $1 \mathrm{~cm}^{3}$ da solựào bissulfito-hirdroquinona (1.3). Honogenizar vigorosamente. 
f) Arrolhar, frouxamente, os tubos ou frascos, : mergalbálos cm agua ferventc, conlida c:m um bíquer, ai permanecendo durants: 10 minutos.

g) A schuir, resirici-los em águis corrente, completanelo-se finaluente o volunse.

h) L'roccelic is leitura colorimetrica.

Cúlculos:

na qual

$$
\mathrm{mg} \text { de fósforo } \mathrm{cm} 100 \mathrm{~cm}^{3} \text { de sôro }=\frac{100 \mathrm{Cc} . \Lambda \mathrm{c} . \mathrm{V}}{\text { Ad.l.S.S. }}
$$

('c = quanliclade de fósforo existente ( $\mathrm{cm} m \underline{m}$ ) no padrĩo;

Ac - altura do combecielo (soluçấo padliño);

$\mathrm{V}=$ volume correspondente à sûma do ácido tricloroncético e sôro, pipetados na operaçũo de desa!luminação;

$A d=$ altura do descunheciclo (filtrado);

$\mathbf{F}=$ volume do filtrado utilizado para o descnvolvimento da côr;

$\mathrm{S}=$ sóro, $\mathrm{cm} \mathrm{cm}^{3}$, tomado para desalbuminar.

Nivlas:

Prolongando-si: 0 acuccimento akim de 10 minutos, produz-se ligciro aumento na intensiclade da cír; êsse fato, porém, nĩo cunstitui inconveniente nas leaturas colorimetricas, porque há proporcionabilidiade en todios os tulsos. A intensidade de côr obtida é nuitos pronunciada c cstável.

MrTODO FOTOMEARICO DI; $R$. S. PEIREIRA (

Soluçöes:

3.1 - Acido sulfúrico Io $\mathrm{N}$.

Mledir $278,5 \mathrm{~cm}^{3}$ de hcido sulfurico, $\mathrm{D}=1,84$ a $15 \circ \mathrm{C}$ e dissolvê-los $\mathrm{cm}$ cêrea de $500 \mathrm{cra}^{3}$ de sigual distilada. Após o resfriamento, proceder it transferincia para matraz de um litro, complctando-sc o volume, depois da necessária laviggem do béquer usiulo na solubilização privia.

Renlizio "A"

3.2 - Molibuato de mocinio a $0,625 \%$ em ácido sulfúrico $5 \mathrm{~N}$.

Dissolver cm um béquer 6,25 g de inolibdato de amónio $\left(\mathrm{Mo}_{7} \mathrm{O}_{24}\left(\mathrm{NH}_{4}\right)_{4}+4 \mathrm{H}_{2} \mathrm{O}\right)$, em cêrea de $400 \mathrm{~cm}$ is de úgua distilada. Transferir para uin frasco volumbtrico de $1000 \mathrm{~cm}^{3}$ c, após acrescentar $500 \mathrm{~cm}^{3}$ de ácido sulfúrico $10 \mathrm{~N}$, completar o volurne com igua distilada. 
3.3 - Reativ' " $B$ "

(oblocar cm frasca escuro, de rôlla estucrilhada, $\breve{g}$ g de virulas de cobre

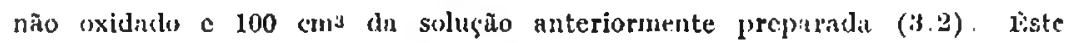
reagente, agitado de quando en quindo, estará fronto para ser utiliwalu, apuis três horiss de contiacto.

3.4 -- Aciclo triclormacition : $10 \%$.

Dissolver etu aigua distilada 50 ge de dicido puríssimo, usando frasco volumétrico sle $500 \mathrm{~cm}^{3}$.

3.5 - Mistura nitro-perclórica.

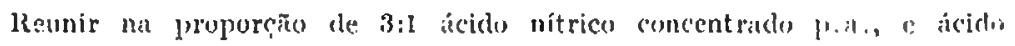
perclúrico paa.

3.6 - Acido sulfúrico $\mathrm{N}$,

Dissolver em um béquer 27,8 cmn de ícido sulfúrico $p . a ., n=1,81$ a IñuC enu cérca de $500 \mathrm{~cm}^{3}$ de água distilada, resfriar e transflerir parn matra\% aferidlo de uin litro, completando-sc o volume.

Anitist:

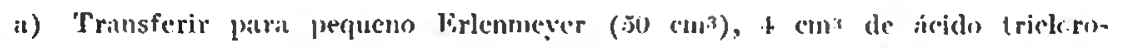
acético a $10 \%$ e sob aggitaçrĭo constante, adicionar, grotejiando, $1 \mathrm{~cm}^{3}$ de sûro.

b) Filtrar, vertenclo de uma sú ve\%, cm pripel de filtro sếco e pràlicaneste isento de cinzas.

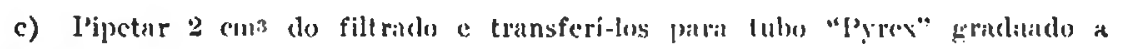
$10 \mathrm{~cm}^{\circ}$.

d) Juntar $2 \mathrm{~cm}^{3}$ de árido sulfúrió $\mathrm{N}$ e atjuecer (cm propuena shamil, onb

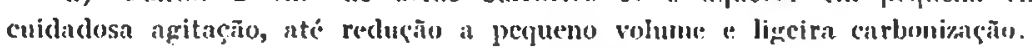

e) Colocar 1-2 gôtas da mislura nitro-perclíriea 3:1 e proceder à mincraliza-

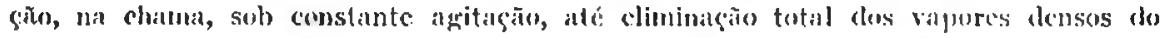
ácido perchórico, alétu dos nitrosos.

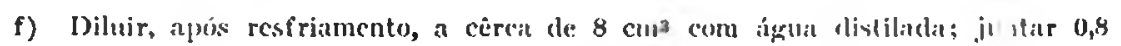
$\mathrm{cm}^{3}$ do reativo " $\Lambda$ " (3.2) e $0,3 \mathrm{~cm}^{3}$ do reativo " 13 " (3.3).

g) Realizar um branco, usando tôdas as soluções e operaudo do mesmer modo.

h) Levar os tubos - desconheciclo e branco - frouxamente arroulhitdos is bitnlıo de água fervente, pelo espaço de 10 minutos.

i) Após resfriamento espontâneo, ato ar, e haver completiclo o volume até a marca, efetuar a lcitura no l'ulf rich, utilizaurdo o filtro S-72.

\section{Cálculo:}

Rcalizadas as leituras no Pulfrich, a dircita e à esquerda, e tomadas us médias da transparência, calcula-se a guantidade de fósforo, pela aplicação da seguinte formula: 
na qual

$$
\text { ing de } P \text { em } 100 \mathrm{cn}^{3} \text { dic sôro }=\frac{0.213 . \mathrm{k} . \mathrm{V}}{\mathrm{Y}}
$$

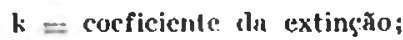

$$
\begin{aligned}
& V=\text { volume em que se desenvolve n côr; } \\
& v=\text { sôro, cm cuns, conticlo na alífuchla tomaclu. }
\end{aligned}
$$

Nolas:

Quando sc concentrar a alífuotir tomada, devc-se trabalhar cuidadosamente com (diamia prefuerna do bico de Bunsen, a fim de se evilarem projeçoes que, subtraindo foisform, crishentomenle, afetariam o resultakle final.

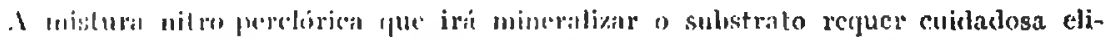

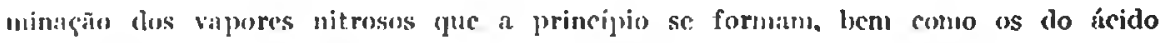
perchirico. Qualguer residuo menor dos mesmos irí falsear os resultados, mercê da inlerferencila cxercida subure o descnvolvinento de côr.

() branco, que servirá como líquido de compensilyău na leitura fotométrica, não deverí tor senão muito fraca tomalidade azul; urna ncentuaçĩo dessa côr indica a necessidade de ser renovado o reagente descrito con $\mathbf{3 . 3}$.

O resfriamcuio espuntânco ao ar, após a permanência pelo espą̧o de 10 minutoss con figua forvents, ic de sumb importancin: os resultados tendem a ser menores, se tal medicha năo forr obserrada.

\section{PARTE EXPERIMENTAL}

Para podermos comparar os resultados obtidos pelos métodos acima descritos, partimos de uma mistura de sôro proveniente de diversos animais, para as. sim dispôrmos de quanlidade suficiente para realizar o objetivo em vista, quer quanto à aplicabilidade, quer quanto ao estudo comparativo.

O sôro foi seprarado duas ou três horas após a sangria, contrariando assim as recomendaçioes de P'erer e Vav Stykz: ( $\left.{ }^{7}\right)$. Segundo êsses autores há um processo de esterificação, que transforma o fósforo inorgânico em orgânico, decorridas duas ou três horas após a colheita, meśno na ausência de hemólise. Ocorre também um processo inverso de hidrólice. com libertação do fósforo contido nas combinações orgânicas, aumentando-se dêsse modo a taxa de fósforo inorgânico.

Como visamos obter o maior volume possível de sôro, não importando, por conseguinte, a riqueza c a origem do fósforo nêle existente, a separaçūo foi feita sem obedecer às normas estabelecidas pelos citados autores.

Convém, entretanto, assinalar a importância que se deve dar à êste ponto, separando o sôro, logo após a colheita, ou então, segundo as verificaçōes de Bur- 
Kens $\left({ }^{8}\right)$, adicionar fluoreto de síclio, na proporção de $20 \mathrm{mg}$ para um rolume. de $10 \mathrm{~cm}^{3}$ de sangue. Este composto previne a transformagīo de fósforo orgânico $\mathrm{cm}$ inorgânico ou vice-versa.

Estas consideracōes nĩo impedem que se lembre c que se opcre. quer na precipitação dos prótidos existentes no sûro, quer na filtragem, pela mancira co. mo foi descrita em cada método, o que se fundamenta em razúes que passamos à expôr: recomendações cabíveis, aliás, à todos os métodos que objelivem a determinaçīo dos fosfatos inorgânicos do sanguc.

O sôro - diz $\mathrm{KoCH}\left({ }^{\circ}\right)$ - que terá seus prótidos removidos por precipitação no ácido tricloroacético à $10 \%$, deverí ser gotejado no volume apropriado dêsse ácido, e não vice-verșa. Tomar-se-á tal precaução, no sentido de prevenir a hidrólise dos ésteres do ácido fosfórico, a quual se processa ràpiclamente, devido à ação dos enzimas. Êstes têm, cutretanto, sua ação inibida, quando operarmos como foi indicado.

A obtenção do fíltrado (do líquiida) proveniente da precipitação dos prótidos no ácido tricloroacélico, - refere ainda Koci $\left({ }^{9}\right)$ - deve ser fcita nela versão de uma só vez no funil filtrante, o que é importante para obviar a libertação do ácido fosföriıo das combinações orgâniças.

Para os métodos de Fiske-Subbarow e Bencdict-Theis utilizamos o colorimetro modêlo Itellige e para o método de R. S. Pereira o folônctro de Pulfrich.

\section{METODO DE FISKE-SUBBAROW}

A fim de observar quais as variaçōes que ocorrem em determinacoócs repctidas no mesmo sôro, fizcmos uma séric de 12 tubos, tendo sido obtidos os resultadus seguintes:

Dosagens procedidas no mesmo nôro

\begin{tabular}{c|c}
\hline Tubos & $\mathrm{mg}$ de $\mathrm{P}$ por $100 \mathrm{~cm}^{3}$ de sôro \\
& \\
1 & 3,66 \\
2 & 3,66 \\
2 & 3,66 \\
4 & 3,62 \\
5 & 3,73 \\
6 & 3,77 \\
7 & 3,71 \\
8 & 3,68 \\
9 & 3,61 \\
10 & 3,60 \\
11 & 3,72 \\
12 & 3,69 \\
\hline
\end{tabular}


A análise estatística (*) dêsses resultados, revela o seguinte:

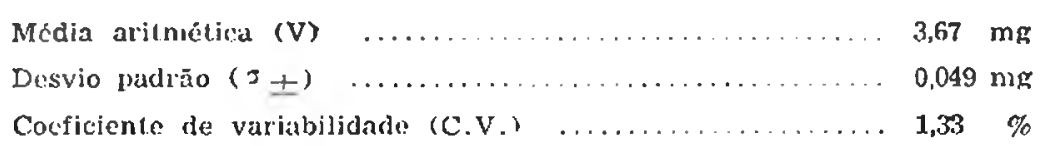

Como se depreende, o cocficicnte de variabilidade, bastante haixo, indica a constîncia dos resullados verificados.

\section{PROVA DE RE,CUPERAÇÃO}

Vịando conhecer a capacidade de recuperacão do método, fizemos outra sériv de luhos, adicionando ao filtrido do sôro, quantidades conlecidas de fósforo. Como se nota no quadro seguinte, foram acrescentadas à volumes fixos do filtrado, quantidades variáveis de uma solução de fosfato.

Fis os resultados:

\begin{tabular}{|c|c|c|c|c|c|}
\hline Tubos & $\begin{array}{l}P \text { (mg) pot } \\
\mathrm{cm}^{3} \text { de sôro }\end{array}$ & $\begin{array}{l}P(\mathrm{mg}) \text { adi- } \\
\text { cionado por } \\
\mathrm{cm}^{3}\end{array}$ & $\begin{array}{c}\mathrm{mg} \text { de } \mathrm{P} \\
\text { (teórico) em } \\
100 \mathrm{~cm}^{3}\end{array}$ & $\begin{array}{c}\mathrm{mg} \text { de } \mathrm{P} \\
\text { (encontrado) } \\
\mathrm{em} 100 \mathrm{~cm}^{3}\end{array}$ & $\begin{array}{l}\text { Porcenta- } \\
\text { gem de re- } \\
\text { cuperação }\end{array}$ \\
\hline 1 & 0,0367 & 0,0207 & 2,87 & 2,87 & 100,00 \\
\hline 2 & 0,0367 & 0,0207 & 2,87 & 2,85 & 99,30 \\
\hline 3 & 0,0367 & 0.0207 & 2,87 & 2,83 & 98,60 \\
\hline 4 & 0,0367 & 0,0248 & 3.07 & 3,11 & 101,30 \\
\hline 5 & 0,0367 & 0,0248 & 3,07 & 3,08 & 100.32 \\
\hline 6 & 0,0367 & 0,0248 & 3,07 & 3,09 & 100,65 \\
\hline 7 & 0,0367 & 0,0290 & 3,28 & 3,27 & 99,69 \\
\hline 8 & 0,0367 & 0,0230 & 3,28 & 3,25 & 99,08 \\
\hline 9 & 0,0367 & 0,0290 & 3,28 & 3,23 & 98,47 \\
\hline 10 & 0,036 ? & 0,0331 & 3,49 & 3,51 & 100,57 \\
\hline 11 & 0,0367 & 0,0331 & 3,49 & 3,44 & 93,56 \\
\hline 12 & 0,0367 & 0.0331 & 3,49 & 3,43 & 98,28 \\
\hline
\end{tabular}

O teste 'T aplicado à recuperaçăo, dá-nos os seguintes resultados:

$$
\begin{aligned}
\mathrm{X} & =0,01416 \\
\mathrm{~S} & =0.009110 \\
12 & =1.50
\end{aligned}
$$

Os valores de: $\mathrm{T}$ nos níveis de $5 \%, 2 \%$ e $1 \%$ são respectivamente, 2,$2 ; 2,72$ e 3,11; o que. em face do resultado encontrado - 1,50 - nos leva a afirmar que a diferença entre $X$ o o seu valor ideal $(0)$ ć atribuivel ao acaso.

(') Consignamos aqui os nonsos agradecimentos ao Prof. Pedro Ekydio de Carvalho, peia -rientação segura imprimida d interpretaçiso estatistica do presente trabalho. 


\section{METODO DE BENEDICT-THEIS *}

Ao acompanharmos a descrição do método, verificamos que os autores não fazem referência alguma acêrca da necessidade ou não de adicionar ao padrão o ácido tricloroacético.

Com tal objetivo, em uma sírie de determinaçōes, foram empregados dois padrōes, tendo sido colocado $\mathrm{cm}$ um dêstes un volume de ácido tricloroacético equivalente ao existente no filtrado de sôro.

Os valores recristrados figuram abaixo:

\begin{tabular}{|c|c|c|}
\hline \multirow{2}{*}{ Tubos } & \multicolumn{2}{|c|}{$\mathrm{mg}$ de fósforo em $100 \mathrm{~cm}^{3}$ de sôro } \\
\hline & $\begin{array}{l}\text { Padrão com } \\
\text { ácido tricloroacético }\end{array}$ & $\begin{array}{l}\text { Padrão sem } \\
\text { ácido tricloroacético }\end{array}$ \\
\hline 1 & 3,78 & 3,75 \\
\hline 2 & 3.71 & 3,68 \\
\hline 3 & 3,63 & 3,68 \\
\hline 4 & 3,67 & 3,62 \\
\hline 5 & 3,61 & 3,57 \\
\hline 6 & 3.61 & $3,5.3$ \\
\hline 7 & 3,71 & 3,70 \\
\hline 8 & 3,61 & 3,62 \\
\hline 9 & 3,69 & 3,70 \\
\hline 10 & 3,76 & 3,70 \\
\hline 11 & 3,69 & 3.61 \\
\hline 12 & 3.59 & 3,51 \\
\hline
\end{tabular}

Resultados estatisticos

\begin{tabular}{|c|c|c|}
\hline & \multicolumn{2}{|c|}{ Padrão } \\
\hline • & $\begin{array}{c}\text { com ácido tricloro- } \\
\text { acético }\end{array}$ & $\begin{array}{c}\text { sem ácido tricloro- } \\
\text { acético }\end{array}$ \\
\hline Média aritmética $(\bar{V}) \ldots \ldots \ldots \ldots$ & $3,67 \mathrm{mg}$ & $3,69 \mathrm{mg}$ \\
\hline Desvio padrão $\left(\sigma_{-1-}\right) \ldots \ldots \ldots \ldots \ldots$ & $0,059 \mathrm{mg}$ & $0,087 \mathrm{mg}$ \\
\hline Coeficient: de variabilidade (C.V) .. & $1,60 \%$ & $2,35 \%$ \\
\hline
\end{tabular}

(*) Em trabalho anterior $\left({ }^{10}\right)$, aplicando o presente milodo ao eletrofotometro de Fisher. obtivemos resultados semelhantes aos aqui consignados. 
Calculando-se o valor do teste $\mathrm{T}$, teremos:

\begin{tabular}{c|c|c}
\hline & teórico & encontrado \\
\hline Valor $t$ ao nivel $1 \% \ldots \ldots \ldots \ldots \ldots \ldots$ & 3,11 & $\begin{array}{c}0,66 \\
\text { não significante }\end{array}$ \\
\hline
\end{tabular}

Ora, o valor 1 an nível de $1 \%$, sendo 3,11 então a diferença por nós encontrada, póde ser imputada ao acaso, o que, em outras palavras, significa que os nossos dados não nos autorizam a concluir ser indispensável a adição do ácido tricloroacélico para compensação da acidês do padráo.

Prosseguindo-se dai e conduzindo-nos dentro do mesmo critério observado no método anterior, fizemos uma série de 12 detcrminações no mesmo sôro, com o intuito de verificar as oscilaçōes possiveis. Nestas dosagens, cmpregamos apenas $4 \mathrm{~cm}^{1}$ do filtrado, equivalendo a $0,8 \mathrm{~cm}^{3}$ do sôro e $3 \mathrm{~cm}^{3} \mathrm{da}$ soluçáo padrão $\left(5 \mathrm{~cm}^{3}=0,03884 \mathrm{mg}\right)$.

Eis os resultados:

Dosagens feltas no mesmo sôro

\begin{tabular}{c|c}
\hline Tubos & $\mathrm{mg}$ de $P$ por $100 \mathrm{~cm}$ ' de sôro \\
\hline 1 & 3,66 \\
2 & 3,65 \\
3 & 3,71 \\
4 & 3,69 \\
5 & 3,66 \\
6 & 3,67 \\
7 & 3,70 \\
8 & 3,63 \\
9 & 3,70 \\
10 & 3,56 \\
11 & 3,74 \\
12 & 3,65 \\
\hline
\end{tabular}

A análise estatística dos resultados cncontrados mostra o seguinte:

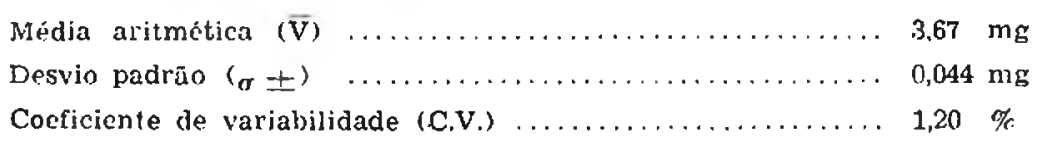




\section{PROVA DE RECUIERACXO}

Seguindo as mesmas normas obervadas no metolo antrior (liske-Subbarow),

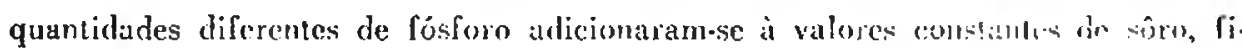
gurando os resultados obtidos pela análise no quasiro sconinte:

\begin{tabular}{|c|c|c|c|c|c|}
\hline Tubos & $\begin{array}{l}\text { P no sôro } \\
\text { por } \mathrm{cm}^{3} \\
(\mathrm{mg})\end{array}$ & $\begin{array}{l}\text { P adiciona- } \\
\text { do por cm: } \\
\text { (mg) }\end{array}$ & $\begin{array}{c}m g \text { de } P \\
\text { (teórico) em } \\
100 \mathrm{~cm}^{3}\end{array}$ & $\begin{array}{c}\text { mg de } P \\
\text { (cncontrado) } \\
\text { cin } 100 \mathrm{~cm} \text { " }\end{array}$ & $\begin{array}{l}\text { Porcenta- } \\
\text { gem de re- } \\
\text { cuperañão }\end{array}$ \\
\hline 1 & $0,0,367$ & 0,0207 & 2.87 & 2,88 & 100,34 \\
\hline 2 & 0,0367 & 0,0207 & 2.87 & 2.83 & 88,60 \\
\hline 3 & 0,0367 & 0,0207 & 2,87 & 2,86 & 99,65 \\
\hline 4 & 0,0367 & 0,0248 & 3,07 & 3,10 & 100,97 \\
\hline 5 & 0,0367 & 0,0248 & 3,07 & 3,15 & 102,60 \\
\hline 6 & 0,0367 & 0,0248 & 3,07 & 3,20 & 104,23 \\
\hline 7 & 0,0367 & 0,0290 & 3,28 & 3,40 & 103,65 \\
\hline 8 & 0,0367 & 0,0290 & 3,28 & 3.37 & 102,74 \\
\hline 9 & 0,0367 & 0.0290 & 3,28 & 3,35 & 102,13 \\
\hline 10 & 0,0367 & 0,0331 & 3,49 & 3,54 & 101,43 \\
\hline 11 & 0,0367 & $0,0.331$ & 3,49 & 3,51 & 100,57 \\
\hline 12 & $0,0: 367$ & 0,0331 & 3,49 & 3.54 & 101,43 \\
\hline
\end{tabular}

()s valores muméricos linites !le t, a quaisquer dos nivei: de $1 \%, 2 \%$ a $5 \%$ sendo menores do (que o encontrado - - 3,38 … e X sendo negativo ( 0,05$)$ isto nos sugere cutão a hipótesé que o mítodo, ao dosar os fosfatos, o faz de lal maneja que implica no aparecimento de um resultado que se afanla da quantirlade real de fosfato existente na amostra,

Além disto. procuramos conhecer as variaçós de fósforo, en função do tempo decorrido após o descuvolvimento da côr, considerando que os aulores e ou tros, nêsse parlicular, däo liberdade ao analista, para conduziram-1ba ao proprio arbitrio.

Para isso, realizamos mais 12 detcrminaçōes e, apús o descuvolvimento da côr, registramos as leituras do colorimetro, observando intervalos de tempo constantes no quadro que se sugue. 


\begin{tabular}{|c|c|c|c|}
\hline \multirow{3}{*}{ Tubos } & \multicolumn{3}{|c|}{$\mathrm{mg}$ de $\mathrm{P}$ em $100 \mathrm{~cm}^{3}$ de sôro } \\
\hline & $A$. & $\mathrm{B}$ & C \\
\hline & logo após & 6 horas & 23 horas \\
\hline 1 & 3,64 & 3,56 & 3,64 \\
\hline 2 & 3.67 & 3,71 & 3,87 \\
\hline 3 & 3,69 & 3,60 & 3,70 \\
\hline 4 & 3,67 & 3,68 & 3.72 \\
\hline 5 & 3,64 & 3,65 & 3,73 \\
\hline 6 & 3,64 & 3,65 & 3,65 \\
\hline 7 & 3,63 & 3,64 & 3,70 \\
\hline 8 & 3,59 & 3,63 & 3,58 \\
\hline 9 & 3,63 & 3,68 & 3,65 \\
\hline 10 & 3,64 & 3,69 & 3,66 \\
\hline 11 & 3,65 & 3,76 & 3,65 \\
\hline 12 & 3,56 & 3.64 & 3,60 \\
\hline
\end{tabular}

A anáiise cstatistica dêsses resultados, manifesta o seguinte:

\begin{tabular}{|c|c|c|c|c|}
\hline & logo após & 6 horas & 23 hor & ras \\
\hline Média aritmética (V) $\ldots \ldots \ldots \ldots$ & $3,63 \mathrm{mg}$ & $3,65 \mathrm{mg}$ & 3,67 & $\mathrm{mg}$ \\
\hline Desvio padraio $(0 \pm 1, \ldots \ldots \ldots \ldots$ & $0,0352 \mathrm{mg}$ & $0.0502 \mathrm{mg}$ & 0,0725 & $\mathrm{mg}$ \\
\hline Coeficiente de variabilidade (C.V.) ... & 0,96 & 1,37 & 1,97 & $\%$ \\
\hline
\end{tabular}

Tendo-se à disposiçăo os dados acima e calculando-se os valores do teste t, resultu:

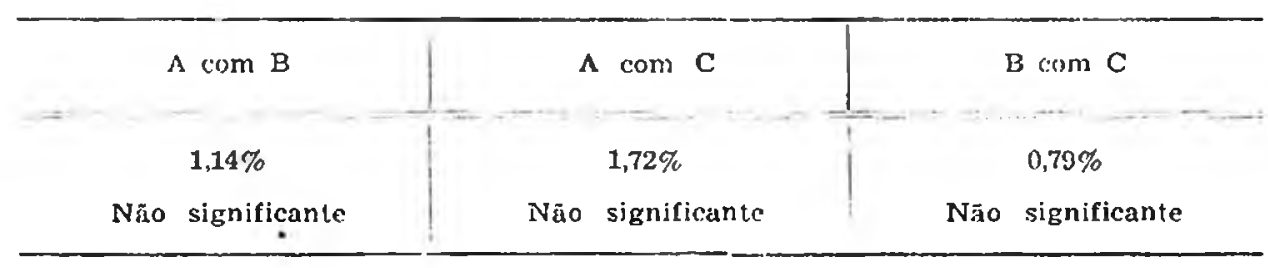


Como vêmos, os nossos dados não hastam para se poder afirmar que o tempo de leitura tenha importância, visto que, difercnģas como as observadas portem ser imputáveis ào acaso, dentro do critério supracitado.

Se as diferenças observadas pudessem ser consideradas significantes, o aumento na média poderia ser atribuível ao padrão que, submetido à luz artificial do colorímetro, por nais tempo, provàvelmente estaria sujeito à uma descoluração maior, em relação ans outros.

METODO FOTOMETRICO DE R. S. PEREIRA (3)

Para o método, trabalhando em amostras do mesmo sôro, obtivemos os rosultados que abaixo vêm tabelados.

\begin{tabular}{c|c}
\hline Tubos & $m g$ de $P$ ex $100 \mathrm{~cm}$ de sôro \\
\hline & \\
1 & 3,72 \\
2 & 3,94 \\
3 & 3,80 \\
4 & 4,24 \\
5 & 3,73 \\
6 & 3,71 \\
7 & 3,63 \\
8 & 3,57 \\
9 & 3,83 \\
10 & 3,71 \\
\hline
\end{tabular}

Da interpretação estatística, resulta:

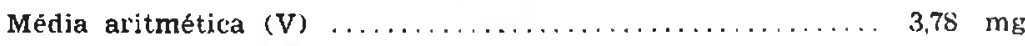

Desvio padrăo $(a)+\ldots \ldots \ldots \ldots \ldots \ldots \ldots \ldots \ldots \ldots, 0,179 \mathrm{mg}$

Coeficiente de variabilidade (C.V.) $\ldots \ldots \ldots \ldots \ldots \ldots \ldots, 4,7,3 \%$

PROVA DE RECUPERAÇÃO

Quanto à recuperação, os resultados poden ser abaixo vistos:

\begin{tabular}{|c|c|c|c|c|c|}
\hline Tubos & $\begin{array}{c}\text { P no sòro } \\
\text { por cm.s } \\
\text { (mg) }\end{array}$ & $\begin{array}{l}\text { P adiciona- } \\
\text { do por cm: } \\
(\mathrm{mg})\end{array}$ & $\begin{array}{c}\text { mg de } P \\
\text { (teórico) } \mathrm{cm} \\
100 \mathrm{~cm}^{3}\end{array}$ & $\begin{array}{c}\text { mg de } P \\
\text { (encontrado) } \\
\mathrm{cm} 100 \mathrm{~cm}^{3}\end{array}$ & $\begin{array}{l}\text { Porcenta- } \\
\text { gem de re- } \\
\text { cuperaçāo }\end{array}$ \\
\hline 1 & 0,0378 & 0,0082 & 2,30 & 2,33 & 101,30 \\
\hline 2 & 0,0378 & 0,0082 & 2,30 & 1,97 & 85,65 \\
\hline 3 & 0,0378 & 0,0082 & 2,30 & 2,17 & 94,34 \\
\hline 4 & 0,0378 & 0,0165 & 2,71 & 2,60 & 95,76 \\
\hline 5 & 0,0378 & 0,0165 & 2,71 & 2,60 & 95,76 \\
\hline 6 & 0,0378 & 0,0165 & 2,71 & 2,71 & 100,00 \\
\hline 7 & 0,0378 & 0,0207 & 2,92 & 2,56 & 87,52 \\
\hline 8 & 0,0378 & 0,0207 & 2,92 & 2,66 & 90,94 \\
\hline 9 & 0,0378 & 0,0207 & 2,92 & 2,79 & 95,38 \\
\hline 10 & 0,0378 & 0,0248 & 3,13 & 3,32 & 106,07 \\
\hline 11 & 0,0378 & 0,0248 & 3,13 & 3,17 & 101,27 \\
\hline 12 & 0,0378 & 0.0248 & 3,13 & 2,88 & 92,01 \\
\hline
\end{tabular}


0 teste $T$, aplicado à recuperação, dá-nos como resultado 2,49 , major do que o valor teórico ao nível de $5 \%(2,20)$, as diferenças observadas náo podendo por éste critério ser atribuidas ao acaso. Vale, entretanto, notar que o valor 2,49 é inferior ans de niveis $2 \%$ e $1 \%$ (2,72 e 3,11 , respectivamente), o que até certo ponto, nos leva a ter cautelas na alirmação da significância da diferença.

\section{DISCUSSÃO}

Coligidos os dados anteriores, temos:

\begin{tabular}{|c|c|c|c|}
\hline & Fiske-Subbarow & Benedict-Theis & R. S. Pereira \\
\hline$M$ (média) $\ldots \ldots \ldots \ldots$ & $3,67 \mathrm{mg}$ & $3,67 \mathrm{mg}$ & $3,78 \mathrm{mg}$ \\
\hline Desvio padrāo $(g \pm) \ldots$ & $0,049 \mathrm{mg}$ & $0,044 \mathrm{mg}$ & $0,179 \mathrm{mg}$ \\
\hline $\begin{array}{l}\text { C.V. (porcentagem de va- } \\
\text { J'iabilidade) } . . . \ldots \ldots \ldots\end{array}$ & $1,33 \%$ & $1,20 \%$ & $4,73 \%$ \\
\hline $\begin{array}{c}N \text { (número de determi- } \\
\text { naçōes) } \ldots \ldots \ldots \ldots \ldots \ldots . . .\end{array}$ & 12 & 12 & 12 \\
\hline
\end{tabular}

$O$ cúlculo do teste ' $\Gamma$, aplicado à recuperação, fornecen os seguintes resultados:

\begin{tabular}{c|c|c|c}
\hline & Fiske-Subbarow & Benedict-Theis & R. S. Pereira \\
\hline \begin{tabular}{c|c|c}
$\mathbf{X}$ \\
$\mathbf{S}$
\end{tabular} & 0,01416 & $-0,05$ & 0,1183 \\
$\mathbf{N}$ & 0,0094108 & 0,0147657 & 0,0474472 \\
Valor de T & $\mathbf{1 , 5 0}$ & $-3,38$ & 2,49 \\
\hline Significáncia & Não significante & Significante & $\begin{array}{c}\text { Significante ao } \\
\text { nivel 5\% }\end{array}$ \\
\hline
\end{tabular}

() teste $F$, cntre os três métodos, dá-nos os seguintes resultados:

\begin{tabular}{c|c|c|c|c}
\hline $\begin{array}{c}\text { Fonte das varia- } \\
\text { çōes }\end{array}$ & $\begin{array}{c}\text { Variaçoes ou sôma } \\
\text { dos quadirados dos } \\
\text { desvios }\end{array}$ & $\begin{array}{c}\text { Gráus de } \\
\text { liberdade }\end{array}$ & Variance & $\begin{array}{c}\text { Valor de F } \\
\text { (calculado) }\end{array}$ \\
\hline $\begin{array}{c}\text { Intcr-detcrmina- } \\
\text { çōes }\end{array}$ & 0,3745 & 31 & 0,01208 & 4,03 \\
$\begin{array}{c}\text { Entre-determina- } \\
\text { ções }\end{array}$ & 0,0954 & 2 & 0,0487 & significante \\
\hline
\end{tabular}


Este resultado, desde que o valor $\mathrm{F}$ ao nivel da distribuigǘn de $5 \%$ rom $\mathrm{n}=2$ e $\left.\mathrm{n}_{2}=3\right]$, i de aproximadamente - 3,3]6 --. diz-110s que os métodos tem variaçôes que pelo critério supra não poden ser alrihuillas an acaso e sim de. correm de fatos que podem on porleriam ser atribuívis à falhas increntes ans métodus.

Como o coeficiente de variabilidade cra lavorável, especialmente no que comcerne aos dois primeiros dos supracitados métodos, resolvemos aplicar o leste de variação aos métodos, tomados dois a dois. Eis o resumo dos cálculos a que essa prática nos levou:

\begin{tabular}{|c|c|c|c|c|c|c|}
\hline Mŕtodo & $\begin{array}{l}\text { Fonte das } \\
\text { variaçõos }\end{array}$ & $\begin{array}{l}\text { Sóma dos } \\
\text { quadra- } \\
\text { dos dos } \\
\text { desvios }\end{array}$ & $\begin{array}{l}\text { Gráus de } \\
\text { liberdade }\end{array}$ & Variance & $\begin{array}{l}\text { Valor de F } \\
\text { calculado }\end{array}$ & $\begin{array}{c}\text { Valor } \\
\text { de } F \text { ao } \\
\text { nivel da } \\
\text { distri- } \\
\text { buis̄o de } \\
5 \%\end{array}$ \\
\hline $\begin{array}{c}\text { Fiske- } \\
\text { Subbarow } \\
\text { e }\end{array}$ & $\begin{array}{l}\text { Intra-deter- } \\
\text { minações }\end{array}$ & 0,0524584 & 22 & $0,002.38447$ & 0,141 & 4,301 \\
\hline $\begin{array}{c}\text { Benedict- } \\
\text { 'Theis }\end{array}$ & $\begin{array}{c}\text { Entrc-deter- } \\
\text { minaçõcs }\end{array}$ & 0,0003375 & 1 & 0,0003375 & $\begin{array}{c}\text { Não } \\
\text { significante }\end{array}$ & \\
\hline $\begin{array}{c}\text { Fiske- } \\
\text { Subbarow }\end{array}$ & $\begin{array}{l}\text { Intra-deter- } \\
\text { minaçöes }\end{array}$ & $0,0.3511$ & 20 & 0.01755 & 3,908 & 4,351 \\
\hline R. S. Pereira & $\begin{array}{l}\text { Entre-deter- } \\
\text { minaçōes }\end{array}$ & 0,0656 & 1 & 0,0686 & $\begin{array}{c}\text { Năo } \\
\text { significante }\end{array}$ & \\
\hline $\begin{array}{c}\text { Bcnedict- } \\
\text { Theis }\end{array}$ & $\begin{array}{l}\text { Inira-deter- } \\
\text { minações }\end{array}$ & 0.3454 & 20 & 0,01727 & 4,522 & \\
\hline e & & & & & & 4,351 \\
\hline R. S. Pereira & $\begin{array}{l}\text { Entre-deter- } \\
\text { minacóes }\end{array}$ & 0,0781 & 1 & 0,0781 & Significante & \\
\hline
\end{tabular}

Os resultados supra nostram claramente que, enquanto a dilerença de variabilidade entre Fiske-Sublarow e Bencdict-Theis é atribuivel ao acaso, o mesmo não acontece em relaçio à Benedict-Theis e R. S. Pereira, em que a diferença é significantc. Este último resultado, aliado à diminuta diferença entre. F' observado e o F teórico no caso de Fiske-Subbarow e $R$. S. Percira, nos sugerc que a diferença de variabilidade notada entre os três méludos deve principalmente correr por conta da discrepância entre o coeficiente de variabilidade do método de R. S. Percira e dos outros dois. 


\section{CONCLUSOES}

Conclui-sc, em face dos resultados da experimentaçīo e dentro dos limites em que: a mesma foi feita, c pelo que a interpretaçäo estatística dos resu!tados nos forncces, ser evidente que o método de Fiske-Subbarow mercer preferéncia ans outros como conseqüencia de maior fidedignidadr des resultados. Quanto aos métodos de Benedict-Theis e IR. S. Pereira, a preferência não é dada cm ra. zîo - o primciro - por ter a sua recuperaģāo e portanto as dosagens, como o comprova o teste $T$, afastadas do teor a ser encontrado; -- o segundo - se hem que somente tendo o teste $\mathrm{T}$ significante ao nivel de $5 \%$, por ter uma percentagem de variabilidade acentuatla relalivamente aos outros - 4,73\% - como aliás o evidencia o teste $F$. Ascim, pois, preconiza-sc na determinação dos fosfalos inorgánicos no sôro de eqiiinos, o método de Fiskc-Subbarow.

\section{RESUMO}

Os autores examinaram a aplicaljilidade dos métodos de Fiskc-Subbarow, Benedict-Theis c R. S. Pereira à determinaçüo do fósforo inorgânico no sangue de cqüinos. Trabalhando com uma única mistura de sôro, realizaram com cada qual dos cilalos métodos uma série de determinaçóes, a lim de observar quais as variaçoes alincrites ì cada um dêles. Visando, depois, conhecer a capacidade de recuperação dos métodos, acrescentaram ao sôro quantidades conhecidas de fósforo, procedendo depois às respectivas determinaçors. Aplicando aos dados obtidos a análise cstatíslica (teste $\mathrm{T}$ e tcste $\mathrm{F}$ ), conclucm os autores que, face zos dados e nas condiçũes da experimentaçāo, merece preferência, pela maior firiedignirlade dos resultados, o método de Fiske-Subbarow. Fncontraram no método de Benedict-Theis a recuperaşão, significantemente ufastada do valor teórico, o que cridencia o teste T. O método de K. S. Percira, cuja variabilidade é. rela. tivanente aos outros, acentuada, tem, - confrontados dois à dois os métodos quanto aos valores da distribuiçăo (teste $\mathrm{F}$ ) - os resultados significantemente afastados do limite máximo da probabilidade, ao nível de $5 \%$.

\section{ABSTRACT}

The AA. examined the applicability of Fiske-Subbarou, Benedicl-Theis and R. S. Pereira methods to the determination of inorganic phosphates in horse blond. Using one single sample of serum, they made a serins of determinations, according to those: methods, in order to observe the variations relative to each one.

Afer this the recovery capacity of the methods was studied by adding to the scrum, known amounts of phosphorus, before respective determinations were male. Statistical analyses of the data obtained (T-test and F-iest), suggests that, under the cxperimental conditions, the Fiske-Subbarow method has to be chosen 
becouse it offers resuls of reliability. The recovery in the Benedict-Theis method wes found of stutistical significance in view of the theoric values to be txpected $(T$-test).

\section{BIBLIOGRAFIA}

1 - Fisk2, C. H. . Stmusuw, Y. - 1925 -... The colorinetric determination of phosphorus. J. Biol. Chem. 66:375-80

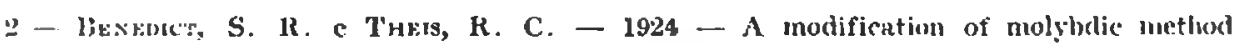
for the determination of inorganic phosphorus in semum. J. Biol. Chem. 61:63-70.

3 - Prrvira, R. S. - 1939 - Determinação espectrofolométricil do iccicle lustórico por

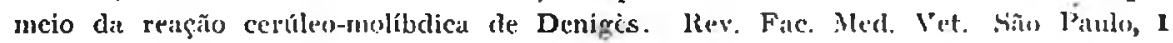
(2) :153-64).

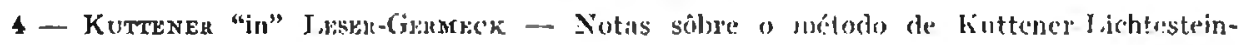
Bodansky, para a determinagiono do fósforn inorgánice no singuc. An. Fac. Med. Sĩo P'aulo, $12(1)$ : :i37-53, 1936.

5 - Folrs, O. - 1930 - Manual Práctico de Análises Biologgicas. Barcelona. Jose Montesó. 1.* edición: 277-81.

6 - Yoe, J. H. - 1928 - I'holometric Chemical Analysis. New York, Jolm Wiley \& Sons, Ine. $1: 310-50$.

7 - Perens, J. I'. e Vax Strks, D. D. -- 1932 - Quantitative dinical chemistry. 'The Williams \& Wilkins (o., 2.

8- Berkexs, J. C. 3. - 1935 - The use of sodium fluoride as a blood anticongulant in blood plosphorus determinations. Bioch. J. 29:796-801.

$9-$ Kocu, F. C. - 1911 - Tractical Methods in Bicchenistry. 3rd.: I62-3. Balltimore, Williams \& Wilkins Company.

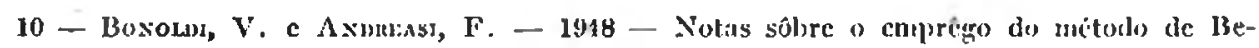
nedict-Theis na determinação do fósforo inorgânico nJ sanguc des edpinos, pelo Elctro-Fotômetro de Fisher. Rev, liac. Mled. Vet. de S'ão l’aulı, 3(t):181-6. 